\title{
EXTREME POINTS AND OUTER FUNCTIONS IN $\boldsymbol{H}^{1}\left(\boldsymbol{U}^{n}\right)$
}

\author{
Kôzô YABUTA
}

(Received March 14, 1970)

It is well known that every extreme point of the unit ball of $H^{1}(U)$ is an outer function with norm 1 and vice versa [1]. In this note we shall point out that for $n \geqq 2$, every outer function (a generalized notion of 1-dimensional outer function, introduced by W.Rudin [2],) with norm 1 is also an extreme point of the unit ball $S$ of $H^{1}\left(U^{n}\right)$, but there exists an extreme point of $S$ which has so many zeros and is consequently not outer. We shall show Theorem 5 for such an example. We state first some facts about extreme points of $S$ and outer functions, which can be shown by a little modification of de LeeuwRudin's method [1].

Let $m_{n}$ denote the Haar measure of the torus $T^{n}$, the distiguished boundary of the unit polydisc $U^{n}$ in the space of $n$ complex variables. If $f$ is holomorphic in $U^{n}$, define

$$
f^{*}(w)=\lim _{r \rightarrow 1} f(r w)
$$

for those $w \in T^{n}$ for which this radial limit exists. A holomorphic function $f \in H^{1}\left(U^{n}\right)$ is said to be outer if

$$
\log |f(0)|=\int_{T^{n}} \log \left|f^{*}(w)\right| d m_{n}(w)
$$

THEOREM 1. Every outer function with norm 1 is an extreme point of $S,(n \geqq 1)$.

THEOREM 2. If $f=g h$, for some non-constant inner function $g$ and $h \in H^{1}\left(U^{n}\right),\|h\|_{1}=1$, then $f$ is not an extreme point of $S,(n \geqq 1)$.

THEOREM 3. A function $f \in H^{1}\left(U^{n}\right)$ lies in the norm closure of the set of all outer functions with norm 1 if and only if $\|f\|_{1}=1$ and $f(z) \neq 0$ for all $z \in U^{n},(n \geqq 1)$.

THEOREM 4. A function $f \in H^{1}\left(U^{n}\right)$ lies in the weak*-closure of the set

We use systematically the notations of [2]. 
of all outer functions with norm 1 if and only if $f \in S$ and $f(z) \neq 0$ for all $z \in U^{n}$, or if $f$ is identically $0,(n \geqq 1)$.

Now we give an example stated above.

THEOREM 5. $\frac{\pi}{4}\left(z_{1}+z_{2}\right)$ is an extreme point of $S,(n \geqq 2)$.

PROOF. We shall show in the case $n=2$ for simplicity of notation, but our proof is general. Assume

$$
z_{1}+z_{2}=\frac{f_{1}\left(z_{1}, z_{2}\right)+f_{2}\left(z_{1}, z_{2}\right)}{2}
$$

where $f_{j} \in H^{1}\left(U^{2}\right),\left\|f_{j}\right\|_{1}=\left\|z_{1}+z_{2}\right\|_{1}=\frac{4}{\pi}(j=1,2)$. Then we have

$$
\begin{aligned}
& \frac{1}{4 \pi^{2}} \int_{0}^{2 \pi} \int_{0}^{2 \pi}\left(\left|f_{1}^{*}\left(e^{i \theta_{1}}, e^{i \theta_{3}}\right)\right|+\left|f_{2}^{*}\left(e^{i \theta_{1}}, e^{i \theta_{2}}\right)\right|\right) d \theta_{1} d \theta_{2} \\
& =\frac{8}{\pi}
\end{aligned}
$$

and

$$
\begin{aligned}
& \frac{1}{2 \pi} \int_{0}^{2 \pi}\left|f_{1}^{*}\left(e^{i \theta_{1}}, e^{i \theta_{2}}\right)+f_{2}^{*}\left(e^{i \theta_{1}}, e^{i \theta_{2}}\right)\right| d \theta_{1} \\
= & \frac{1}{2 \pi} \int_{0}^{2 \pi}\left|2\left(e^{i \theta_{1}}+e^{i \theta_{2}}\right)\right| d \theta_{1} \\
= & \frac{8}{\pi} \quad \text { a. e. } \theta_{2} \in(0,2 \pi) .
\end{aligned}
$$

Hence there is a measurable set $E_{1}$ of $m E_{1}=1$ such that

$$
\begin{aligned}
& \frac{1}{2 \pi} \int_{0}^{2 \pi}\left(\left|f_{1}^{*}\left(e^{i \theta_{1}}, e^{i \theta_{2}}\right)\right|+\left|f_{2}^{*}\left(e^{i \theta_{1}}, e^{i v 2}\right)\right|\right) d \theta_{1} \\
= & \frac{8}{\pi} \quad\left(\theta_{2} \in E_{1}\right) .
\end{aligned}
$$


On the other hand, there is a measurable set $E_{2}$ of $m E_{2}=1$ such that

$$
f_{j}\left(z_{1}, e^{i \theta_{2}}\right) \in H^{1}(U) \quad\left(j=1,2, \theta_{2} \in E_{2}\right)
$$

and

$$
\lim _{r_{1} \rightarrow 1} f_{j}\left(r_{1} e^{i \theta_{1}}, e^{i \theta_{2}}\right)=f_{j}^{*}\left(e^{i \theta_{1}}, e^{i \theta_{2}}\right) \text { a.e. } \theta_{1}\left(\theta_{2} \in E_{2}, j=1,2\right)
$$

Set $E=E_{1} \cap E_{2}$ and $\left\|f_{j}\left(z_{1}, e^{i \theta_{2}}\right)\right\|_{1}=\alpha_{j}\left(\theta_{2}\right), j=1,2, \theta_{2} \in E$.

Then, by (3), we have $\alpha_{1}\left(\theta_{2}\right)+\alpha_{2}\left(\theta_{2}\right)=\frac{8}{\pi}$.

Fix $\theta_{2} \in E$. Assume $\alpha_{1}\left(\theta_{2}\right) \leqq \alpha_{2}\left(\theta_{2}\right)$ and put

$$
\begin{gathered}
g_{1}\left(z_{1}\right)=f_{1}\left(z_{1}, e^{i \theta_{2}}\right)+\frac{\alpha_{2}-\alpha_{1}}{2 \alpha_{2}} f_{2}\left(z_{1}, e^{i \theta_{2}}\right) \\
g_{2}\left(z_{1}\right)=f_{2}\left(z_{1}, e^{i \theta_{2}}\right) \frac{\alpha_{1}+\alpha_{2}}{2 \alpha_{2}} .
\end{gathered}
$$

Then we have

$$
\left\|g_{j}\left(z_{1}\right)\right\|_{1} \leqq \frac{\alpha_{1}+\alpha_{2}}{2}=\frac{4}{\pi} \quad(j=1,2)
$$

By assumption we have

$$
\begin{aligned}
\frac{g_{1}\left(z_{1}\right)+g_{2}\left(z_{1}\right)}{2} & =\frac{f_{1}\left(z_{1}, e^{i \theta_{2}}\right)+f_{2}\left(z_{1}, e^{i \theta_{2}}\right)}{2} \\
& =z_{1}+e^{i \theta_{2}} .
\end{aligned}
$$

Since $\left\|z_{1}+e^{i \theta_{2}}\right\|_{1}=\frac{4}{\pi}$, and $z_{1}+e^{i \theta_{2}}$ is an outer function in $H^{1}(U)$ and thus an extreme point of $H^{1}(U)$, we must have

$$
g_{j}\left(z_{1}\right)=z_{1}+e^{i \boldsymbol{o}_{2}} \quad(j=1,2) .
$$


We have, therefore,

$$
f_{j}\left(z_{1}, e^{i \boldsymbol{\theta}_{2}}\right)=\frac{\pi}{4} \alpha_{j}\left(\theta_{2}\right)\left(z_{1}+e^{i \boldsymbol{\theta}_{2}}\right) \quad(j=1,2) .
$$

These expressions hold also for $\alpha_{1}>\alpha_{2}$, and so for all $\theta_{2} \in E$.

Now there is a measurable set $E_{3}$ of $m E_{3}=1$ and $\theta_{1} \in(0,2 \pi)$ such that

$$
f_{1}\left(e^{i \theta_{1}}, z_{2}\right) \in H^{1}(U)
$$

and

$$
\begin{aligned}
& \lim _{r_{1} \rightarrow 1} \lim _{r_{2} \rightarrow 1} f_{1}\left(r_{1} e^{i \theta_{1}}, r_{2} e^{i \theta_{2}}\right) \\
& =\lim _{r_{2} \rightarrow 1} \lim _{r_{1} \rightarrow 1} f_{1}\left(r_{1} e^{i \theta_{1}}, r_{2} e^{i \theta_{2}}\right) \\
& =f_{1}^{*}\left(e^{i \theta_{1}}, e^{i \theta_{2}}\right) \quad\left(\theta_{2} \in E_{3}\right) .
\end{aligned}
$$

Using this fact and (4), we extend $\alpha_{1}\left(\theta_{2}\right)$ holomorphically into $U=\left\{z_{2}:\left|z_{2}\right|<1\right\}$ by

$$
f_{1}\left(e^{i \theta_{1}}, z_{2}\right)=\frac{\pi}{4} \alpha_{1}\left(z_{2}\right)\left(e^{i \theta_{1}}+z_{2}\right)\left(z_{2} \in U\right)
$$

Since $e^{i \theta_{1}}+z_{2}$ is an outer function and $f\left(e^{i \theta_{1}}, z_{2}\right) \in H^{1}(U), \alpha_{1}(z)$ lies in $N_{*}(U)$. And as $0 \leqq \alpha_{1}\left(\theta_{2}\right) \leqq 8 / \pi, \alpha_{1}(z)$ lies in $H^{\infty}(U)$ and hence must be constant. By definition of $\alpha_{1}\left(\theta_{2}\right)$ and $\alpha_{2}\left(\theta_{2}\right)$, we must have

$$
\alpha_{1}=\alpha_{2}=\frac{4}{\pi}
$$

which shows via $(4)$ that $f_{1}=f_{2}=z_{1}+z_{2}$. This proves that $\frac{\pi}{4}\left(z_{1}+z_{2}\right)$ is an extreme point of the unit ball of $H^{1}\left(U^{2}\right)$. Q. E. D.

\section{REFERENCES}

[1] K. DE LEeUW AND W. Rudin, Extreme points and extremum problems in $H_{1}$, Pacific J 
Math., 8(1958), 463-485.

[2] W. RuDIN, Function theory in Polydiscs, Benjamin, 1969.

[ 3 ] A. ZYGMUND, Trigonometric series II, Cambridge University Press, 1959.

MATHEMATICAL INSTITUTE

TÔHOKU UNIVERSITY

SENDAI, JAPAN 